\title{
Characterization of microRNA expression in primary human colon adenocarcinoma cells (SW480) and their lymph node metastatic derivatives (SW620)
}

This article was published in the following Dove Press journal: OncoTargets and Therapy

Wei Yan

Wenchao Yang

Zhongcai Liu

Guoyang Wu

Department of General Surgery, Zhongshan Hospital, Xiamen University, Xiamen, Fujian 36/004,

People's Republic of China
Correspondence: Guoyang Wu Department of General Surgery, Zhongshan Hospital, Xiamen University, Hubin South Road 20I-209, Siming District, Xiamen 361004, Fujian, People's Republic of China Emailw13400677II6@aliyun.com
Background and objective: Metastasis is the major cause of cancer-related deaths in patients with colon cancer, however, the exact molecular mechanism is unclear. MicroRNAs (miRNAs) play an important role in the pathogenesis and progression of cancer. Therefore, in this study, we aimed to identify differentially expressed miRNAs in two colon carcinoma cell lines: SW480, derived from primary colon carcinoma and SW620, derived from lymph node metastasis, which were obtained from the same patient.

Materials and methods: Three independent samples of cancer cells were collected from SW480 and SW620 cells, respectively. An miRNA microarray platform, miRCURY LNA ${ }^{\mathrm{TM}}$ microRNA array with 1,223 probes containing 3,000 capture probes, was used to determine the miRNA expression profiles of these two cell lines. Differentially expressed miRNAs were validated by quantitative reverse transcription-polymerase chain reaction (qRT-PCR).

Results: The raw data were submitted to the Gene Expression Omnibus database (GSE72412). Thirteen miRNAs were differentially expressed between SW480 and SW620 cells, of which, seven miRNAs (hsa-miR-920, hsa-miR-636, hsa-miR-766-3p, hsa-miR-545-5p, hsa-miR195-3p, hsa-miR-125a-3p, and hsa-miR-196b-3p) were found to be upregulated and six miRNAs (hsa-miR-3613-3p, hsa-miR-29b-3p, hsa-miR-1297, hsa-miR-141-5p, hsa-miR-200c-3p, and hsa-miR-141-3p) were found to be downregulated. Target analysis of the predicted miRNAs showed that these genes were primarily involved in protein binding, cell adhesion, and cancer metastasis. Furthermore, qRT-PCR validated the results of miRNA microarray.

Conclusion: This is the first systematic analysis of the differences of miRNAs between SW480 and SW620 cells. The results provide useful information to explore potential biomarkers of miRNAs for predicting colon cancer metastasis.

Keywords: microRNA, colon cancer, metastasis, microarray

\section{Introduction}

Colon cancer is one of the most common types of cancers and metastasis is the leading cause of death in patients with colon cancer. ${ }^{1}$ However, the molecular mechanism involved in the metastasis is not yet clear. Many studies demonstrate that microRNAs (miRNAs) play an important role in the pathogenesis and progression of cancer. ${ }^{2}$ miRNAs are small noncoding RNAs that suppress target gene expression at the posttranscriptional level by binding to the $3^{\prime}$-untranslated region of the target mRNA. ${ }^{3}$ Although miRNAs have been found to promote or suppress tumor metastasis, the exact role of miRNAs in colon cancer metastasis remains to 
be uncovered. Functional studies indicate that miRNAs have important functions in tumor progression, especially recurrence, drug resistance, and metastasis. ${ }^{4}$ Majority of the researchers have provided strong evidence that miRNAs play an important role during tumorigenesis and tumor progression. $^{5-8}$

With this background that the miRNAs are involved in tumor development and progression, in this study, we hypothesized that miRNAs also play a key role in colon cancer metastasis. The metastatic potential of SW620 cells, derived from lymph node metastasis, is known to be higher than that of SW480 cells, derived from primary colon cancer tissues of the same patient, which have been used as model cell lines for analyzing the metastatic ability. ${ }^{9,10}$ Therefore, we hypothesized that the genes expressed, such as miRNAs, which differ between SW480 and SW620 cells, could be related to tumor metastasis. Until now, there are no systematic reports with respect to the miRNA expression profile of the two recognized cell lines with distinct metastatic capacity. In this study, we profiled the miRNAs of the SW620 and SW480 colon cancer cell lines.

\section{Materials and methods \\ Cell culture}

The human colon cancer cell lines SW480 and SW620 were purchased from the American Tissue Culture Collection (Manassas, VA, USA) and were routinely maintained in RPMI-1640 medium (HyClone, Logan, UT, USA) supplemented with 10\% fetal bovine serum (Thermo Fisher Scientific, Waltham, MA, USA), $100 \mathrm{U} / \mathrm{mL}$ penicillin sodium and $100 \mathrm{mg} / \mathrm{mL}$ streptomycin sulfate at $37^{\circ} \mathrm{C}$ in a humidified air atmosphere containing $5 \% \mathrm{CO}_{2}$. Cells were used in logarithmic growth phase.

\section{RNA extraction}

Total RNA was isolated using TRIzol (Thermo Fisher Scientific) and purified with RNeasy mini kit (Qiagen) according to manufacturer's instructions. RNA quality and quantity were measured by using NanoDrop spectrophotometer (ND-1000; NanoDrop Technologies, Thermo Fisher Scientific, Waltham, MA, USA) and RNA integrity was determined by gel electrophoresis.

\section{Labeling of miRNA and array hybridization}

RNA labeling and array hybridization were performed according to Exiqon's manual. First, after quality control, the miRCURY LNA ${ }^{\mathrm{TM}} \mathrm{Hy} 3^{\mathrm{TM}} / \mathrm{Hy} 5^{\mathrm{TM}}$ Power labeling kit
(Exiqon, Vedbaek, Denmark) was used according to the manufacturer's guideline for miRNA labeling by following steps: 1) $1 \mu \mathrm{L}$ RNA in $2.0 \mu \mathrm{L}$ of water was combined with $1.0 \mu \mathrm{L}$ of calf intestinal alkaline phosphatase (CIAP) buffer and CIAP (Exiqon). The mixture was incubated for $30 \mathrm{~min}$ at $37^{\circ} \mathrm{C}$; 2) the reaction was terminated by incubation for $5 \mathrm{~min}$ at $95^{\circ} \mathrm{C}$. Then, $3.0 \mu \mathrm{L}$ of labeling buffer, $1.5 \mu \mathrm{L}$ of fluorescent label (Hy3 ${ }^{\mathrm{TM}}$ ), $2.0 \mu \mathrm{L}$ of dimethyl sulfoxide, and $2.0 \mu \mathrm{L}$ of labeling enzyme were added into the mixture. The labeling reaction was incubated for $1 \mathrm{~h}$ at $16^{\circ} \mathrm{C}$; and 3) the labeling reaction was terminated by incubation for $15 \mathrm{~min}$ at $65^{\circ} \mathrm{C}$. Then, the Hy3 ${ }^{{ }^{\text {TM}}}$-labeled samples were hybridized on the miRCURY LNA ${ }^{\mathrm{TM}}$ microRNA Array (v.18.0) (Exiqon) according to the array manual steps as follows: 1) the total $25 \mu \mathrm{L}$ mixture from Hy3 $3^{\text {TM}}$-labeled samples with $25 \mu \mathrm{L}$ hybridization buffer was first denatured for $2 \mathrm{~min}$ at $95^{\circ} \mathrm{C}$ and then incubated on ice for $2 \mathrm{~min} ; 2$ ) then, hybridized on the microarray for $16-20 \mathrm{~h}$ at $56^{\circ} \mathrm{C}$ in a 12 -Bay Hybridization Systems (Hybridization System - Nimblegen Systems, Inc., Madison, WI, USA); and 3) following hybridization, the slides were prepared and washed several times using wash buffer kit (Exiqon). Eventually, the slides were scanned using the Axon GenePix 4000B microarray scanner (Axon Instruments, Foster City, CA, USA).

\section{Data analysis}

The raw data were submitted to the Gene Expression Omnibus database (GSE72412) (http://www.ncbi.nlm.nih.gov/ geo/). The scanned images were then imported into GenePix Pro 6.0 software (Axon Instruments) for grid alignment and data extraction. The replicated miRNAs were averaged and those with intensities $\geq 30$ in all samples were chosen to calculate the normalization factor. The data expressed were normalized using the median normalization. After normalization, significant differentially expressed miRNAs between the two groups were identified using fold change and $p$-value. Differentially expressed miRNAs between the two samples were filtered through fold change. Finally, hierarchical clustering was performed to show distinguishable miRNA expression profiling among samples.

\section{Quantitative reverse transcription- polymerase chain reaction ( $\mathrm{qRT}$ RCPR)}

Cells of human colon cancer cell lines SW480 and SW620 in the logarithmic growth period were collected. Following the isolation of total RNA, cDNA were synthesized using a miDETECT A Track ${ }^{\mathrm{TM}}$ miRNA qRT-PCR Starter kit (RIOBIO Biotechnology Co., Ltd., Guangzhou, People's 
Republic of China). All the miRNAs' primers (miDETECT A Track ${ }^{\mathrm{TM}}$ miRNA qPCR Primer Set), including hsa-miR920, hsa-miR-636, hsa-miR-766-3p, hsa-miR-545-5p, hsa-miR-195-3p, hsa-miR-125a-3p, hsa-miR-196b-3p, hsa-miR-3613-3p, hsa-miR-29b-3p, hsa-miR-1297, hsamiR-141-5p, hsa-miR-200c-3p, and hsa-miR-141-3p, and control primer (miDETECT A Track ${ }^{\mathrm{TM}}$ U6 qPCR Primer Set) were also purchased. We performed qRT-PCR on an ABI 7500 real-time PCR system (Thermo Fisher Scientific) according to the manufacturer's protocol by miDETECT A Track ${ }^{\mathrm{TM}}$ miRNA qRT-PCR Kit (RIOBIO Biotechnology Co., Ltd.). Dissociation curve analysis was used to verify the generation of a single product. U6 was used as a reference gene, and all reactions were repeated three times. Data were analyzed using the ABI 7500 Prism sequence detection software (Thermo Fisher Scientific) and the relative expression was calculated using the $2^{-\Delta \Delta \mathrm{Cq}}$ method.

\section{Statistical analysis}

Statistical analysis was performed using SPSS statistical software (version 17.0; SPSS, Inc., Chicago, IL, USA). The significant difference between the two groups was analyzed by one-way analysis of variance using paired $t$-test. Data are expressed as mean \pm standard deviation. A $p$-value $<0.05$ was considered as a significant difference.

\section{Results \\ miRNA expression profiles between SW620 and SW480 cells}

To identify the difference in miRNA expression between SW620 and SW480 cells, we used the miRNA microarray platform, miRCURY LNATM microRNA Array with 1,223 probes containing 3,000 capture probes, and screened differentially expressed miRNAs. First, three independent cell samples were collected from SW480 and SW620 cells, respectively. Total RNA were extracted from the total six samples (SW480-1, SW480-2, SW480-3, SW620-1, SW620-2, and SW620-3). For spectrophotometer, the ratio of optical density (OD) (A260/A280) should be close to 2.0 for pure RNA (ratios between 1.8 and 2.1 are acceptable). The OD A260/A230 ratio should be more than 1.8. We validated the RNA quantification and assure the quality of RNA (Figure 1). Furthermore, we obtained a good quality control chip distribution after chip hybridization: 1) The probe distribution of each chip between six total RNAs of samples is not consistent before standardization, and the median of each chip is at the same level and the distribution of the probe is

\begin{tabular}{lllllll}
\hline A Sample ID & $\begin{array}{l}\text { OD260/280 } \\
\text { ratio }\end{array}$ & $\begin{array}{l}\text { OD260/230 } \\
\text { ratio }\end{array}$ & Conc $(\mathbf{n g} / \mathbf{\mu L})$ & Volume $(\boldsymbol{\mu L})$ & Quantity (ng) & $\begin{array}{l}\text { QC result } \\
\text { pass or fail }\end{array}$ \\
\hline SW480-1 & 2.04 & 2.09 & $2,155.72$ & 140 & $301,800.80$ & Pass \\
SW480-2 & 2.02 & 2.09 & $2,458.42$ & 140 & $344,178.80$ & Pass \\
SW480-3 & 2.04 & 2.10 & $2,214.00$ & 140 & $309,960.00$ & Pass \\
SW620-1 & 2.05 & 2.23 & $1,915.59$ & 130 & $249,026.70$ & Pass \\
SW620-2 & 2.04 & 2.15 & $2,191.88$ & 140 & $306,863.20$ & Pass \\
SW620-3 & 2.04 & 2.12 & $2,111.91$ & 150 & $316,786.50$ & Pass \\
\hline
\end{tabular}

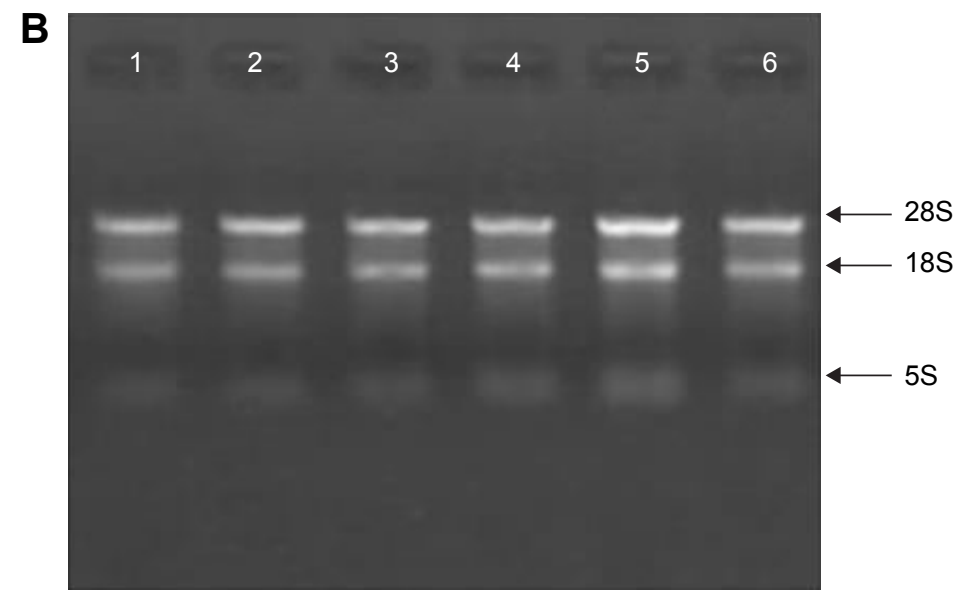

Figure I RNA quality control in total six cell samples. (A) RNA quantification and quality assurance by NanoDrop ND- 1000. (B) RNA integrity and gDNA contamination test by denaturing agarose gel electrophoresis. Lanes I, 2, 3, 4, 5, and 6: total RNA of samples SW480-I, SW480-2, SW480-3, SW620-I, SW620-2, and SW620-3. Abbreviation: OD, optical density. 

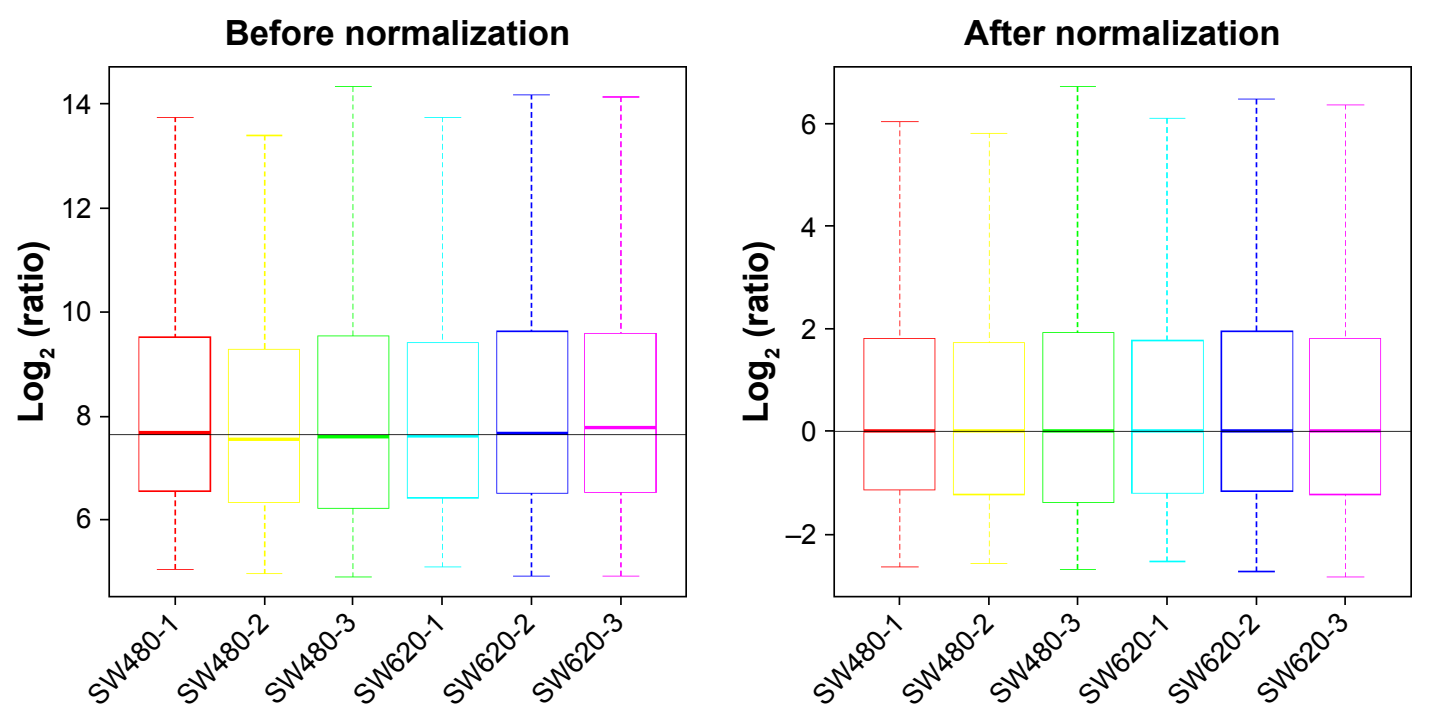

Figure 2 The distribution of the probe on the chip between six total RNAs of samples SW480-I, SW480-2, SW480-3, SW620-I, SW620-2, and SW620-3. The box plot reflects the distribution of the probe on the chip, which can be used to compare the effect of the probe before and after the standardization. The probe distribution of each chip is not consistent before standardization, and the median of each chip is at the same level and the distribution of the probe is close after the middle value standardization.
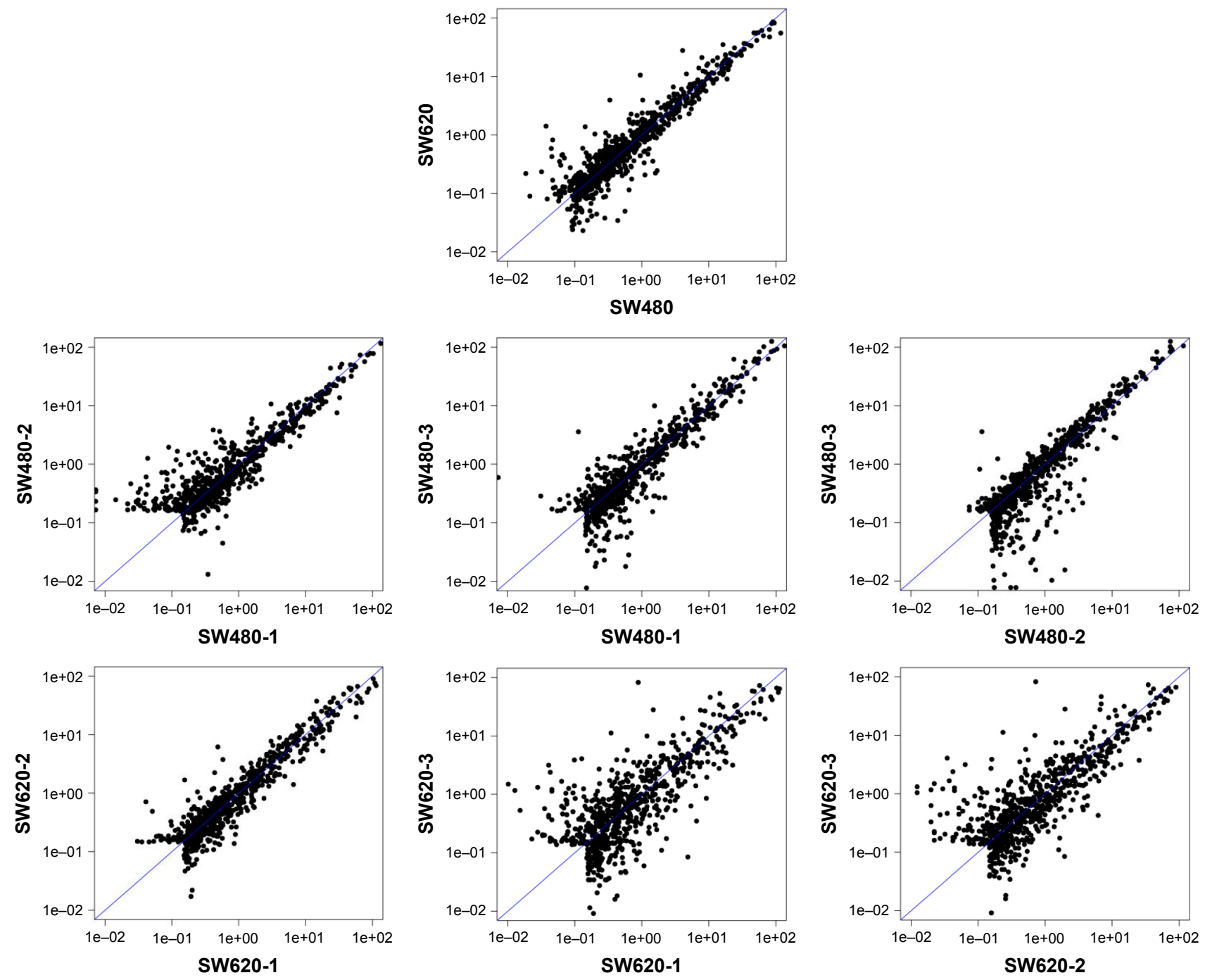

Figure 3 The distribution of the probe on the chip (scatter plot) between six total RNAs of samples SW480-I, SW480-2, SW480-3, SW620-I, SW620-2, and SW620-3. The scatter plot reflects the repeatability between the chips or the groups. The better the repeatability is, the closer the diagonal line is. 
Table I Pearson correlation coefficient to measure repeatability between six total RNAs of samples SW480-I, SW480-2, SW480-3, SW620-I, SW620-2, and SW620-3

\begin{tabular}{|l|l|l|l|}
\hline \multicolumn{5}{|l}{ Correlation coefficient matrix } \\
\hline & SW480-I & SW480-2 & SW480-3 \\
\hline SW480-I & I & 0.9764724 & $0.95 I 095 I$ \\
\hline SW480-2 & 0.9764724 & I & $0.974 I 08$ \\
\hline SW480-3 & $0.95 I 095 I$ & $0.974 I 08$ & I \\
\hline & & & \\
\hline & SW620-I & SW620-2 & SW620-3 \\
\hline & I & 0.933746 & 0.81 I2635 \\
\hline SW620-I & 0.933746 & I & $0.838343 I$ \\
\hline SW620-3 & $0.8 I I 2635$ & $0.838343 I$ & I \\
\hline & & & \\
\hline & SW480 & SW620 & \\
\hline SW480 & I & 0.9546237 & \\
\hline SW620 & 0.9546237 & I & \\
\hline
\end{tabular}

Notes: The closer to I the correlation coefficient is, the better the repeatability is

close after the middle value standardization (Figure 2);2) The scatter plot reflects the repeatability between the chips or the groups. The better the repeatability is, the closer the diagonal line is (Figure 3); 3) The closer to 1 the correlation coefficient is, the better the repeatability is (Table 1). Eventually, we found that 13 differentially expressed miRNAs (including seven upregulated [hsa-miR-920, hsa-miR-636, hsa-miR766-3p, hsa-miR-545-5p, hsa-miR-195-3p, hsa-miR-125a-3p, and hsa-miR-196b-3p] and six downregulated known miRNAs [hsa-miR-3613-3p, hsa-miR-29b-3p, hsa-miR-1297, hsa-miR-141-5p, hsa-miR-200c-3p, and hsa-miR-141-3p], respectively) in the SW620 cells in contrast to the SW480 cells. Figure 4 and Table 2 summarize the 13 miRNAs that show the significant differences.

\section{Validation of differentially expressed miRNAs}

miRNA-specific qRT-PCR was performed to validate the differentially expressed miRNAs. The expression levels of hsa-miR-920, hsa-miR-636, hsa-miR-766-3p, hsa-miR-545-5p, hsa-miR-195-3p, hsa-miR-125a-3p, and hsa-miR-196b-3p were found to be significantly upregulated in SW620 (Figure 5A), whereas those of hsa-miR-3613-3p, hsa-miR-29b-3p, hsa-miR-1297, hsa-miR-141-5p, hsa-miR200c-3p, and hsa-miR-141-3p were found to be downregulated (Figure 5B). These results agree with the results of the miRNA microarray analysis.

\section{Analysis of the predicted target genes of differentially expressed miRNAs}

To investigate the potential function of predicted target genes, we analyzed the differentially expressed miRNAs by searching previously published studies regarding the particular genes'
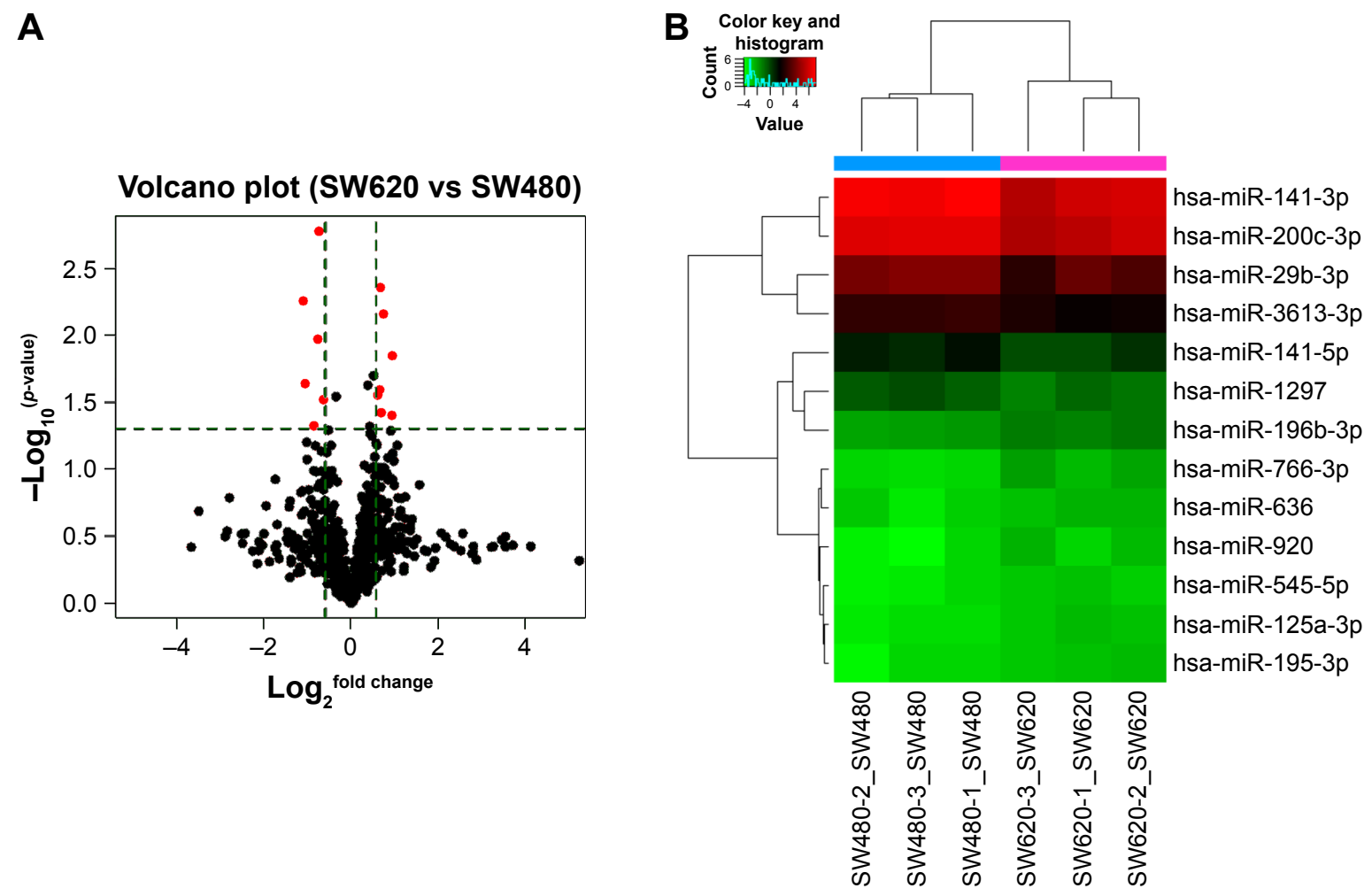

Figure 4 Significantly differentially expressed miRNAs in SW620 cells compared with SW480 cells. (A) Volcano plot: I 3 red points represent the I 3 significantly differentially expressed miRNAs according to fold change $>1.5$ and $p<0.05$. (B) Heatmap and hierarchical clustering of 13 significantly differentially expressed miRNAs. Abbreviation: miRNA, microRNA. 
Table 2 The list of differentially expressed miRNAs between SW620 cells and SW480 cells

\begin{tabular}{llll}
\hline miRNAs & Fold change & Type of regulation & $p$-value \\
\hline hsa-miR-920 & 1.930594 & $U_{p}$ & 0.039586 \\
hsa-miR-636 & 1.53241 & $U_{p}$ & 0.027953 \\
hsa-miR-766-3p & 1.939967 & $U_{p}$ & 0.014147 \\
hsa-miR-545-5p & 1.587454 & $U_{p}$ & 0.025508 \\
hsa-miR-195-3p & 1.62515 & $U_{p}$ & 0.037874 \\
hsa-miR-125a-3p & 1.605793 & $U_{p}$ & 0.004376 \\
hsa-miR-196b-3p & 1.686959 & Up & 0.006909 \\
hsa-miR-36I3-3p & 1.663110 & Down & 0.039586 \\
hsa-miR-29b-3p & 2.066123 & Down & 0.001667 \\
hsa-miR-1297 & 1.550251 & Down & 0.022966 \\
hsa-miR-14I-5p & 1.796777 & Down & 0.030168 \\
hsa-miR-200c-3p & 1.695634 & Down & 0.047333 \\
hsa-miR-14I-3p & 2.133825 & Down & 0.010694 \\
\hline
\end{tabular}

Notes: $p<0.05$, fold change $>1.5$.

Abbreviation: miRNA, microRNA.

functions. We could predict tumor-associated gene targets of the significantly differentially expressed 13 miRNAs. Analysis of predicted miRNAs' targets showed that these genes were primarily involved in protein binding, cell adhesion, and cancer metastasis. Tables 3 and 4 summarize the results.

\section{Discussion}

In this study, we used SW480 and SW620 cell lines that have different metastatic capabilities (Table 5) and we hypothesized that miRNAs that differ between SW480 and SW620 cells could be related to the formation of cancer metastasis. Therefore, we investigated the miRNA expression profiling between SW480 and SW620 cells using an miRCURY LNA ${ }^{\mathrm{TM}}$ miRNA array platform and identified some differentially expressed miRNAs between them. According to our results, seven
miRNAs were found to be upregulated and six were found to be downregulated in SW620 cells in contrast to SW480 cells, whose functions are known to be closely associated with tumor metastasis. Thirteen differentially expressed miRNAs (including seven upregulated [hsa-miR-920, hsa-miR-636, hsa-miR-766-3p, hsa-miR-545-5p, hsa-miR-195-3p, hsa-miR125a-3p, and hsa-miR-196b-3p] and six downregulated known miRNAs [hsa-miR-3613-3p, hsa-miR-29b-3p, hsa-miR-1297, hsa-miR-141-5p, hsa-miR-200c-3p, and hsa-miR-141-3p], respectively) were identified and qRT-PCR was performed to validate the array results. Furthermore, we analyzed our results on the miRNAs with target genes and found that many target genes are related to tumor progression and metastasis in previously published peer-reviewed literature.

Chen et al found that the risk allele for rs16405 disrupted a binding site for human miR-920 which would negatively regulate beta-transducin repeat-containing protein (betaTrCP) and proposed an miR-920-mediated betaTrCP regulation model depending on rs16405 genotype, which may influence the risk of hepatocellular carcinoma (HCC). The molecular mechanism was, at least in part, found to be hsa-miR-920-mediated regulation, which is possibly involved in the pathogenesis of HCC. ${ }^{11}$ Jang et al revealed that miR-636 might function as a tumor suppressor miRNA affecting $\mathrm{HCC}$ tumorigenesis via downregulation of Ras, and that ANT2 suppression by shRNA could exert an anticancer effect by restoring the expression of miR-636 in HCC. ${ }^{12}$ Some studies demonstrate that targeting DNMT3B by miR-766 and other miRNAs, decreased methylation of tumor suppressor genes, such as SFRP1, SFRP2, WIF1, and DKK2, and returned the expression of these tumor suppressor genes
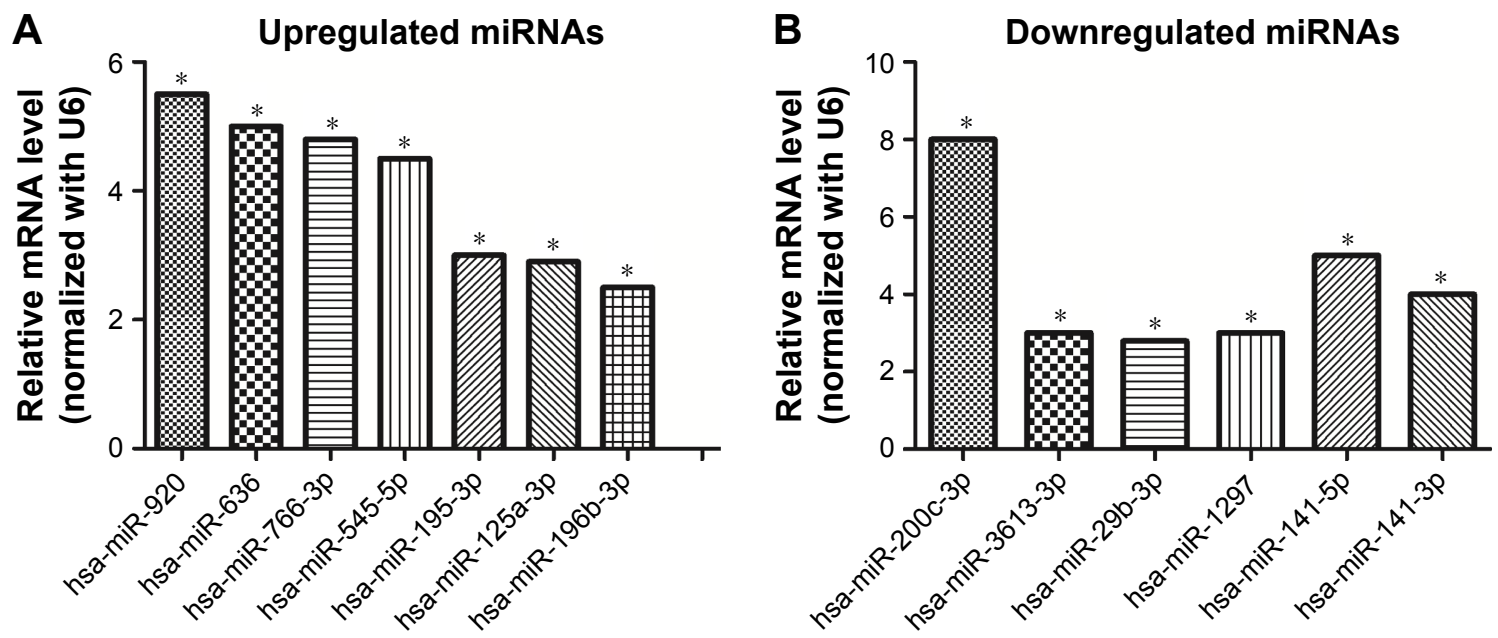

Figure 5 Validation of miRNA microarray results by qRT-PCR. (A) Seven upregulated miRNAs in SW620 cells compared with SW480 cells. (B) Six downregulated miRNAs in SW620 cells in contrast to SW480 cells. U6 was used as a normalization control. Data represent the mean \pm standard deviation of three biological replicates. *p $<0.05$. Abbreviations: miRNA, microRNA; qRT-PCR, quantitative reverse transcription-polymerase chain reaction. 
Table 3 Putative target genes of the seven upregulated miRNAs

\begin{tabular}{lll}
\hline miRNAs & Target gene & Molecular function \\
\hline hsa-miR-920 & betaTrCP & Involved in cancer pathogenesis \\
hsa-miR-636 & Ras, ANT2 & Tumorigenesis \\
hsa-miR-766-3p & SF2, DNMT3B, SIRT6 & Cancer progression \\
hsa-miR-545-5p & RIG-I, cyclin DIa, CDK4 & Cell proliferation, invasion \\
hsa-miR-195-3p & PVTI, Notch, FGF-I8, SMAD7, TLR2, & Cell proliferation, migration, and invasion and apoptosis, angiogenesis \\
& YAPI, GABARAPLI & and autophagy, cell colony formation abilities, remodeling of the \\
hsa-miR-125a-3p & THP-I, NFI, Bcl-2, foxp3, E2F2, ERRalpha & extracellular matrix, and inflammatory pathway mediators \\
hsa-miR-196b-3p & P65, Meis2, and PPP3CC & Cell differentiation and apoptosis and cancer progression \\
\hline
\end{tabular}

Abbreviation: miRNA, microRNA.

Table 4 Putative target genes of the six downregulated miRNAs

\begin{tabular}{lll}
\hline miRNAs & Target gene & Molecular function \\
\hline hsa-miR-36I3-3p & MCPIPI, CTLI, SLC44AI & Cell proliferation \\
hsa-miR-29b-3p & SIRTI, MCL-I, DNMT3B, CDC42, & Regulates oxidative stress, cell transformation, EMT, metastasis, \\
& TETI, Akt3, VEGF, c-Myc & cell migration and invasion, and angiogenesis/tumorigenesis \\
hsa-miR-1297 & HMGA2, EZH2, Cox-2, TRIB2, & Associated with metastasis of cancer, cell proliferation, \\
& C/EBPalpha, PTEN & invasion, and apoptosis \\
hsa-miR-14I-5p & FUS, EWS, BMII, HOTAIR & Tumor growth and metastasis, cell proliferation and migration \\
hsa-miR-200c-3p & c-Myc, MSN, MAD2LI, KIM-I, & Cellular proliferation, migration, and invasiveness, regulates \\
& BMII, ZEBI-ASI, LUCATI, Foxf2 & cancer progression, EMT, and drug resistance \\
hsa-miR-I4I-3P & SKA2, GABI, ZEB2, HGFR & Tumor growth and metastasis \\
\hline
\end{tabular}

Abbreviations: miRNA, microRNA; EMT, epithelial-mesenchymal transition.

Table 5 Information about SW620 and SW480 cell line from American Tissue Culture Collection (ATCC)

\begin{tabular}{lll}
\hline & SW620 & SW480 \\
\hline ATCC Number & CCL-227 TM & CCL-228 ${ }^{\text {TM }}$ \\
Link address & http://www.atcc.org/Products/All/CCL-227.aspx & http://www.atcc.org/Products/All/CCL-227.aspx \\
Organism & Homo sapiens, human & Homo sapiens, human \\
Tissue & Colon; derived from metastatic site: lymph node & Colon \\
Morphology & Epithelial & Epithelial \\
Culture properties & Adherent & Adherent \\
Disease & Dukes' type C, colorectal adenocarcinoma & Dukes' type B, colorectal adenocarcinoma \\
Age & 5 I years & 50 years \\
Gender & Male, Caucasian & Male, Caucasian \\
Tumorigenic & Yes & Yes \\
Effects & Tumors developed within 2I days at I00\% frequency (5/5) & Tumors developed within 2I days at I00\% frequency (5/5) \\
Applications & in nude mice inoculated subcutaneously with I0 (7) cells & in nude mice inoculated subcutaneously with I0 (7) cells \\
\hline
\end{tabular}

which can contribute to lethal effect on colon cancer cells and reducing tumorigenicity of these cells. ${ }^{13}$ In addition, miR-766-3p can promote cell proliferation by targeting the expression of SF2 in renal cell carcinoma (RCC), which is significantly associated with poor survival in a large cohort of RCC specimens. ${ }^{14}$ miR-545 is found to be involved in tumor proliferation and progression and is associated with overall survival and progression-free survival in patients with cancer. ${ }^{15-17}$ Furthermore, miR-195 is associated with the progression of fibrosis and remodeling of the extracellular matrix, which can regulate cell proliferation, migration, angiogenesis, and autophagy. ${ }^{18-20}$ In addition, miR-125a may represent a novel therapeutic target for the treatment of osteosarcoma and some findings have suggested that the detected miR-125a-5p levels in plasma could be used as a potential biomarker in early Kawasaki disease diagnosis..$^{21,22}$ The activation of miR-125a confers resistance to $\mathrm{BRAF}(\mathrm{V})(600 \mathrm{E})$ melanoma cells to BRAF inhibitors. ${ }^{23}$ Furthermore, miR196b significantly contributes to leukemia development, ${ }^{24}$ and miR-29b could inhibit glioma cell migration and invasion 
and inhibit breast cancer cell angiogenesis. ${ }^{25,26}$ miR-1297 inhibits the growth and metastasis of colorectal cancer. ${ }^{27}$ miR-141 functions as a tumor suppressor in colorectal cancer SW480 cells, ${ }^{28}$ and miR-200c regulates cancer progression, epithelial-mesenchymal transition, and drug resistance..$^{29,30}$

\section{Conclusion}

In conclusion, we used miRNA microarray technology to analyze the different miRNA expression profiling between SW620 and SW480 cells and found that 13 miRNAs were significantly deregulated. The expression levels of miRNAs were validated by qRT-PCR, and the results were found to be consistent with the results detected by microarray analysis. Target gene predictions indicated that differentially expressed miRNAs play an important role in the metastasis of colon cancer. However, further study on these miRNAs and their functions in the tumorigenesis of colon cancer is necessary.

\section{Acknowledgments}

This work was supported by National Nature Science Foundation of China (Grant No 81172285) and Natural Science Foundation of Fujian Province, People's Republic of China (Grant No 2018J01392 and 2018J01390).

\section{Disclosure}

The authors report no conflicts of interest in this work.

\section{References}

1. Wasserman I, Lee LH, Shia J, et al. SMAD4 loss in colorectal cancer: correlation with recurrence, chemoresistance, and immune infiltrate. J Clin Oncol. 2017;35(Suppl 4):587.

2. Lian W, Stoletov K, Jovel J, et al. Development of a miRNA-based signature to predict human cancer metastasis. Cancer Res. 2017; 77(Suppl 13):1044.

3. Farh KK, Grimson A, Jan C, et al. The widespread impact of mammalian microRNAs on mRNA Repression and Evolution. Science. 2005; 310(5755):1817-1821.

4. Pencheva N, Tavazoie SF. Control of metastatic progression by microRNA regulatory networks. Nat Cell Biol. 2013;15(6):546-554.

5. Wu CW, Storey KB. Regulation of Smad mediated microRNA transcriptional response in ground squirrels during hibernation. Mol Cell Biochem. 2018;439(1-2):151-161.

6. Zhou J, Hu M, Wang F, Song M, Huang Q, Ge B. miR-224 controls human colorectal cancer cell line HCT116 proliferation by targeting Smad4. Int J Med Sci. 2017;14(10):937-942.

7. Rupaimoole R, Slack FJ. MicroRNA therapeutics: towards a new era for the management of cancer and other diseases. Nat Rev Drug Discov. 2017;16(3):203-222.

8. Li B, Xu WW, Han L, et al. MicroRNA-377 suppresses initiation and progression of esophageal cancer by inhibiting CD133 and VEGF. Oncogene. 2017;36(28):3986-4000.

9. Tanaka S, Hosokawa M, Yonezawa T, Hayashi W, Ueda K, Iwakawa S. Induction of epithelial-mesenchymal transition and down-regulation of miR-200c and miR-141 in oxaliplatin-resistant colorectal cancer cells. Biol Pharm Bull. 2015;38(3):435-440.
10. Leibovitz A, Stinson JC, McCombs WB 3rd, McCoy CE, Mazur KC, Mabry ND. Classification of human colorectal adenocarcinoma cell lines. Cancer Res. 1976;36(12):4562-4569.

11. Chen SG, He Y, Ding J, et al. An insertion/deletion polymorphism in the $3^{\prime}$ untranslated region of $\beta$-transducin repeat-containing protein (betaTrCP) is associated with susceptibility for hepatocellular carcinoma in Chinese. Biochem Biophys Res Commun. 2010;391(1):552-556.

12. Jang JY, Lee YS, Jeon YK, Lee K, Jang JJ, Kim CW. ANT2 suppression by shRNA restores miR-636 expression, thereby downregulating Ras and inhibiting tumorigenesis of hepatocellular carcinoma. Exp Mol Med. 2013;45(1):e3.

13. Afgar A, Fard-Esfahani P, Mehrtash A, et al. MiR-339 and especially miR-766 reactivate the expression of tumor suppressor genes in colorectal cancer cell lines through DNA methyltransferase 3B gene inhibition. Cancer Biol Ther. 2016;17(11):1126-1138.

14. Chen C, Xue S, Zhang J, et al. DNA-methylation-mediated repression of miR-766-3p promotes cell proliferation via targeting SF2 expression in renal cell carcinoma. Int J Cancer. 2017;141(9):1867-1878.

15. Gu Y, Zhang M, Peng F, et al. The BRCA1/2-directed miRNA signature predicts a good prognosis in ovarian cancer patients with wild-type BRCA1/2. Oncotarget. 2015;6(4):2397-2406.

16. Song B, Ji W, Guo S, et al. miR-545 inhibited pancreatic ductal adenocarcinoma growth by targeting RIG-I. FEBS Lett. 2014;588(23): 4375-4381.

17. Du B, Wang Z, Zhang X, et al. MicroRNA-545 suppresses cell proliferation by targeting cyclin D1 and CDK4 in lung cancer cells. PLoS One. 2014;9(2):e88022.

18. Wu D, Li Y, Zhang H, et al. Knockdown of Lncrna PVT1 enhances radiosensitivity in non-small cell lung cancer by sponging Mir-195. Cell Physiol Biochem. 2017;42(6):2453-2466.

19. Mo J, Zhang D, Yang R. MicroRNA-195 regulates proliferation, migration, angiogenesis and autophagy of endothelial progenitor cells by targeting GABARAPL1. Biosci Rep. 2016;36(5):e00396.

20. Du J, Zheng R, Xiao F, et al. Downregulated MicroRNA-195 in the bicuspid aortic valve promotes calcification of valve interstitial cells via targeting SMAD7. Cell Physiol Biochem. 2017;44(3):884-896.

21. Tao T, Shen Q, Luo J, et al. MicroRNA-125a regulates cell proliferation via directly targeting E2F2 in osteosarcoma. Cell Physiol Biochem. 2017;43(2):768-774.

22. Li Z, Jiang J, Tian L, et al. A plasma mir-125a-5p as a novel biomarker for Kawasaki disease and induces apoptosis in HUVECs. PLoS One. 2017;12(5):e0175407

23. Koetz-Ploch L, Hanniford D, Dolgalev I, et al. MicroRNA-125a promotes resistance to BRAF inhibitors through suppression of the intrinsic apoptotic pathway. Pigment Cell Melanoma Res. 2017;30(3): 328-338.

24. Popovic R, Riesbeck LE, Velu CS, et al. Regulation of mir-196b by MLL and its overexpression by MLL fusions contributes to immortalization. Blood. 2009;113(14):3314-3322.

25. Shi C, Ren L, Sun C, et al. miR-29a/b/c function as invasion suppressors for gliomas by targeting CDC42 and predict the prognosis of patients. Br J Cancer. 2017;117(7):1036-1047.

26. Li Y, Cai B, Shen L, et al. MiRNA-29b suppresses tumor growth through simultaneously inhibiting angiogenesis and tumorigenesis by targeting Akt3. Cancer Lett. 2017;397:111-119.

27. Wang Y, Xue J, Kuang H, Zhou X, Liao L, Yin F. microRNA-1297 inhibits the growth and metastasis of colorectal cancer by suppressing cyclin D2 expression. DNA Cell Biol. 2017;36(11):991-999.

28. Long ZH, Bai ZG, Song JN, et al. miR-141 inhibits proliferation and migration of colorectal cancer SW480 cells. Anticancer Res. 2017;37(8):4345-4352.

29. Mutlu M, Raza U, Saatci Ö, Eyüpoğlu E, Yurdusev E, Şahin Ö. miR-200c: a versatile watchdog in cancer progression, EMT, and drug resistance. J Mol Med (Berl). 2016;94(6):629-644.

30. Hur K, Toiyama Y, Takahashi M, et al. MicroRNA-200c modulates epithelial-to-mesenchymal transition (EMT) in human colorectal cancer metastasis. Gut. 2013;62(9):1315-1326. 
OncoTargets and Therapy

\section{Publish your work in this journal}

OncoTargets and Therapy is an international, peer-reviewed, open access journal focusing on the pathological basis of all cancers, potential targets for therapy and treatment protocols employed to improve the management of cancer patients. The journal also focuses on the impact of management programs and new therapeutic agents and protocols on

perspectives such as quality of life, adherence and satisfaction. The manuscript management system is completely online and includes a very quick and fair peer-review system, which is all easy to use. Visit http://www.dovepress.com/testimonials.php to read real quotes from published authors.

Submit your manuscript here: http://www.dovepress.com/oncotargets-and-therapy-journal 